\title{
OPTIMIZING THE SECOND-ORDER OPTICAL NONLINEARITIES OF ORGANIC MOLECULES: ASYMMETRIC CYANINES AND HIGHLY POLARIZED POLYENES
}

\author{
Seth R. Mardera,b, Christopher B. Gorman, ${ }^{a}$ Lap-Tak Cheng ${ }^{c}$ and Bruce G. Tiemann ${ }^{a, b}$ \\ a: Jet Propulsion Laboratory, California Institute of Technology, \\ 4800 Oak Grove Drive, Pasadena, CA 91109, USA
}

b: The Beckman Institute, California Institute of Technology, Pasadena, CA, 91125, USA

c: E. I. DuPont de Nemours and Company, Inc., Wilmington, DE 19880-0328, USA

\begin{abstract}
$\underline{\text { ABSTRACT }}$
We recently reported that there is an optimal combination of donor and acceptor strengths for a given molecular length and bridge structure that maximizes $|\beta|$. For this combination, there is the correct degree of bond length alternation and asymmetry in the molecule. Our recent findings suggest that molecules that can be viewed as asymmetric cyanines with relatively small amounts of bond length alternation are nearly optimal. In this manner, we have identified molecules with nonlinearities many times that of conventional chromophores for a given length. In this paper, we will present a new computational analysis that allows the correlation of bond length alternation with hyperpolarizabilities and will present EFISH data on simple donoracceptor polyene chromophores.
\end{abstract}

\section{INTRODUCTION}

The realization of efficient nonlinear optical (NLO) devices requires optimization of various materials properties, including transparency, ease of fabrication, orientational, photochemical and thermal stability as well as nonlinear optical susceptibility. The rational design of optimized materials can be facilitated by establishing a fundamental understanding of the chemical structure/property relationships that govern molecular hyperpolarizabilities. Beyond facilitating the search for highly nonlinear chromophores, this understanding will be helpful when considering tradeoffs between nonlinearity and the aforementioned secondary materials properties necessary to optimize device performance. In this paper we discuss a computational method that predicts the structural features of molecules that have maximized linear polarizabilities $(\alpha)$, first $(\beta)$, or second $(\gamma)$ hyperpolarizabilities for a given molecular length, and present the results of electric field induced second-harmonic generation (EFISH) studies for a class of molecules that exhibit large $\beta$. Throughout, we will emphasize what we believe to be a key, experimentally accessible structural parameter, bond length 
alternation, and illustrate how it can be correlated with linear and nonlinear optical response.

Prototypical organic NLO chromophores contain a polarizable $\pi$-electron system with donor and acceptor groups to create an asymmetric molecular environment. It has been recently hypothesized that there is an optimal combination of donor/acceptor strengths that maximizes $\beta .^{1}$ This analysis was based upon a two-state model for $\beta$ derived from perturbation theory ${ }^{2-4}$ in which the dominant contribution to $\beta$ arises from the ground-state and a single charge transfer excited-state. In this model, $\beta$ is proportional to:

$$
\left(\mu_{\mathrm{ee}}-\mu_{\mathrm{gg}}\right)\left(\frac{\mu_{\mathrm{ge}}^{2}}{E_{\mathrm{ge}}^{2}}\right)
$$

where $g$ labels the ground-state, $e$ labels the charge transfer excited-state, and $\mu$ is the dipole matrix element between the subscripted states. The terms in this equation, clockwise from left, are proportional to (1) the dipole moment change between the two states, (2) the square of the transition dipole moment between the two states and, (3) the inverse square of the charge transfer (CT) transition energy. Most molecules previously explored have dominantly aromatic ground-states; the corresponding electron transfer states have predominantly quinonal ring structures. Electronic polarization, arising from an applied electric field, results in a perturbed ground-state wave function with increased quinonal character and decreased resonance stabilization energy (Figure 1a). Thus, aromaticity in the ground-state impedes electronic polarization in an applied field and effectively reduces the effective donor and acceptor strengths of a given pair connected by an aromatic bridge.

$\mathrm{a}$

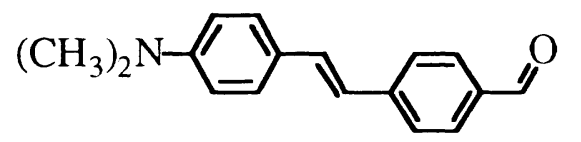

$\mathrm{b}$

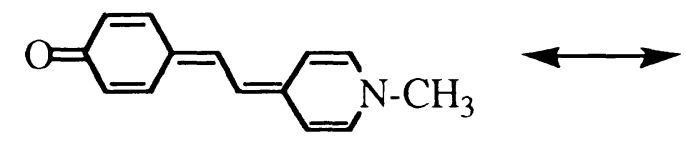

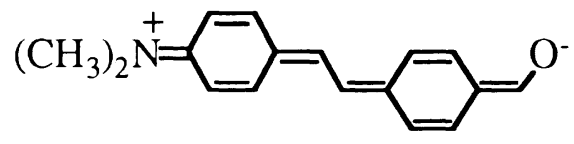
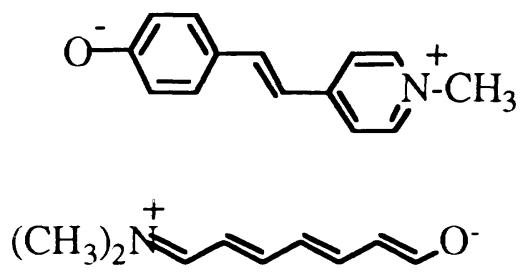

Figure 1. Limiting resonance forms for molecules with (a) an aromatic ground-state where resonance energy is lost in the CT-state, (b) a quinonal ground-state where resonance energy is gained in the CT-state, and (c) a polyene ground-state where resonance energy does not change in the CT-state. 


\section{EXTERNAL ELECTRIC FIELD-DEPENDENT COMPUTED HYPERPOLARIZABILITIES}

The visible absorption maximum and extinction coefficients of merocyanines (Figure $1 \mathrm{~b}$ and 1c) are sensitive to the dielectric properties of the surrounding medium. Although solvatochromic behavior is usually interpreted solely as a change in the electronic distribution of a molecule at a fixed nuclear geometry, ${ }^{5}$ for merocyanines it has been shown that the molecular geometry also undergoes significant changes. ${ }^{6-12}$ Thus, in more polar solvents, merocyanines exhibit a change in bond length alternation and a larger ground-state dipole moment $(\mu)$. Furthermore, by varying the strength of the donor and acceptor end groups of merocyanine molecules, the degree of groundstate polarization and bond length alternation can be tuned. Since one can systematically tune their degree of ground-state polarization and bond length alternation, merocyanines are of particular interest as model systems for developing NLO structure/property relationships. Computational analyses that ignore their medium-dependent chemical structure, accordingly, will tend to underestimate the charge-transfer contribution to their ground-state wave function in most solvents 7 leading to calculated hyperpolarizabilities that can differ significantly with those measured in solution.

We sought to develop a computational procedure to systematically vary the groundstate polarization of a molecule and observe the resulting changes in bond length alternation, $\alpha, \beta$, and $\gamma$. Ideally, we would like to be able to accurately describe the geometry and electronic structure of various donor/acceptor molecules in solvent environments of varying polarity. Currently, several research groups are developing procedures to simulate the effects of solvent upon polarizable molecules. ${ }^{13}$ When these techniques have been developed, it will be of great interest to apply them to hyperpolarizability calculations. One presently available way to polarize a molecule is to apply an external electric field and permit the molecule to assume a new equilibrium geometry and electronic configuration. Although the polarizations in a molecule created by donors and acceptors or by solvent stabilization of charge-separation are not strictly analogous to polarization created by an external electric field, we will show that this method qualitatively reproduces experimental trends in geometry and polarizabilities as a function of increasing ground-state polarization.

Using the AM1 parameterization in the MOPAC package ${ }^{14,} 15$ we examined several prototypical merocyanines $(\mathbf{1}, \mathbf{2}, 3)$ and a cyanine (4) under the influence of an external perturbation designed to vary the ground-state polarization and geometry. This perturbation was two positive and two negative point charges (Sparkles) for 1, 2, and $\mathbf{3}$ and a single negative Sparkle for 4 , moved in steps from $40 \AA$ to $4 \AA$ from the molecule as is shown in Figures 2 and 3. At each fixed Sparkle distance, the geometry was optimized, and $\mu, \alpha, \beta$ and $\gamma$ were calculated by a finite field subroutine ${ }^{16}$ in the presence of the Sparkles. It is important to note that although two explicit fields are applied to the molecule, the point charge field and the electric field within the finite field calculation, 
these fields have distinctly different effects on the molecule. In particular, the first field is designed to polarize the molecule and is permitted to influence both its geometric and electronic structure. Thus, at the start of the finite field computation, the molecule is in a new equilibrium geometry and electronic configuration, within which the hyperpolarizabilities are then calculated. Using Sparkles to polarize the electrons without permitting the molecular geometry to change results in a finite field calculation of a molecule in a non-equilibrium, polarized, state. Likewise, removal of the Sparkles after geometry optimization, but before the finite field procedure leaves nuclei in a position corresponding to a polarized molecule, but removes the potential that stabilizes the polarization. Both of the above procedures were performed; however, for each case, since the nuclei and the electrons were not in equilibrium configurations, much larger applied fields were needed to polarize the molecule, as compared to applying a field and allowing the geometry to optimize. These results suggested to us that both the applied electric field and the position of the nuclei make important contributions to the polarization response and that the most reasonable procedure was to account for both effects. By varying the strength of the electric field employed in the finite field routine, we avoided both numerical accuracy problems and electron configurational changes, observed for field strengths that are too small and too large, respectively.

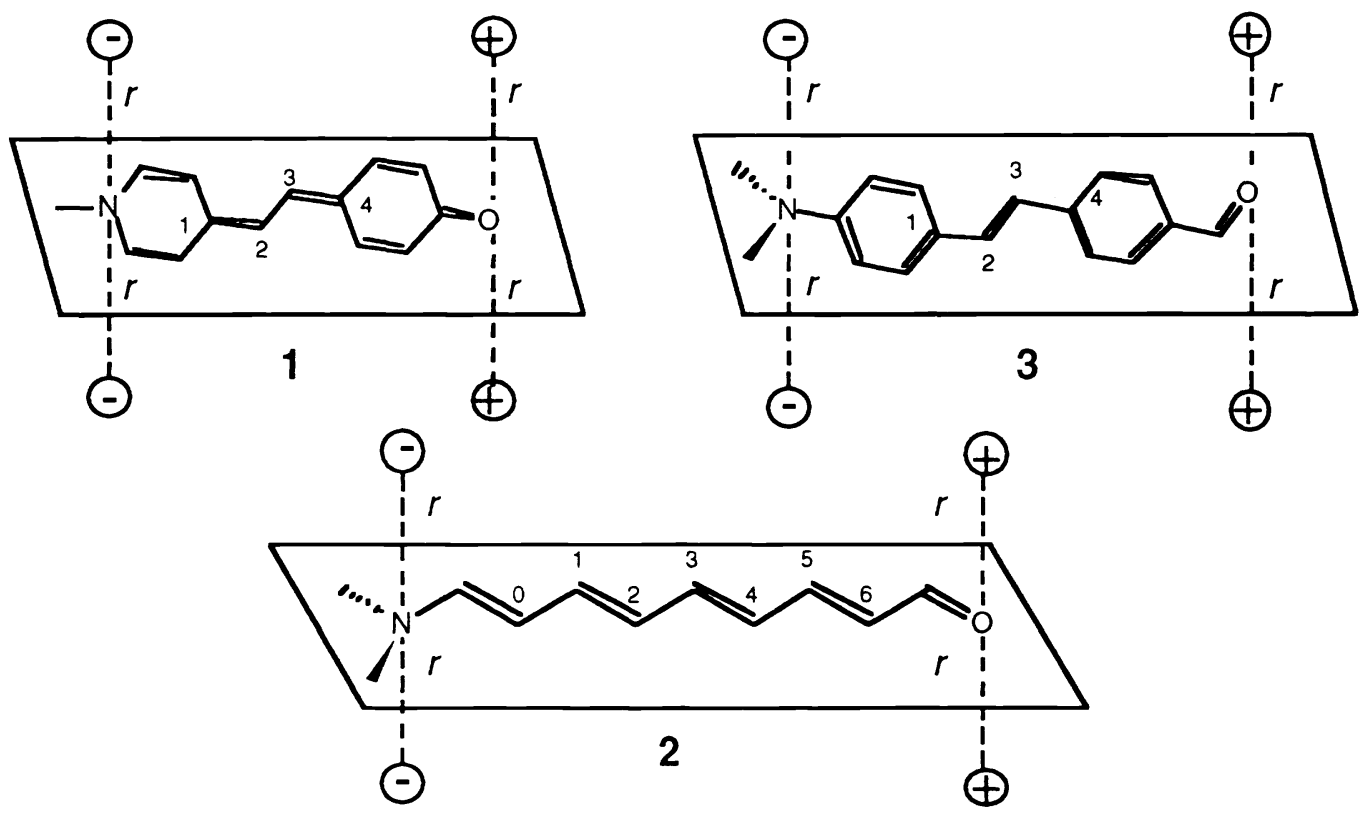

Figure 2. The structures of molecules 1 through 3 represented in their the neutral resonance structures. The Sparkles lie perpendicular to the plane of the molecule at a distance $(r)$ from the nitrogen and oxygen atoms. Bond length alternation (BLA) is defined so that a large negative value results for the molecule in its neutral form. For 1 , $\mathrm{BLA}=\left(\mathrm{r}\left(\mathrm{C}_{1}-\mathrm{C}_{2}\right)+\mathrm{r}\left(\mathrm{C}_{3}-\mathrm{C}_{4}\right)\right) / 2-\mathrm{r}\left(\mathrm{C}_{2}-\mathrm{C}_{3}\right)$. For $2 \mathrm{BLA}=\left(\mathrm{r}\left(\mathrm{C}_{1}-\mathrm{C}_{2}\right)+\mathrm{r}\left(\mathrm{C}_{3}-\mathrm{C}_{4}\right)+\mathrm{r}\left(\mathrm{C}_{5}-\mathrm{C}_{6}\right)\right) / 3-$ $\left(r\left(\mathrm{C}_{0}-\mathrm{C}_{1}\right)+r\left(\mathrm{C}_{2}-\mathrm{C}_{3}\right)+\mathrm{r}\left(\mathrm{C}_{4}-\mathrm{C}_{5}\right)\right) / 3$. For 3, BLA $=r\left(\mathrm{C}_{2}-\mathrm{C}_{3}\right)-\left(\mathrm{r}_{1}\left(\mathrm{C}_{1}-\mathrm{C}_{2}\right)+\mathrm{r}\left(\mathrm{C}_{3}-\mathrm{C}_{4}\right)\right) / 2$. $r\left(\mathrm{C}_{\mathrm{a}}-\right.$ $\left.\mathrm{C}_{\mathrm{b}}\right)$ is the distance in $\AA$ between carbon atoms a and $\mathrm{b}$. 
As noted earlier, polarization by point charges is a crude equivalent to that achieved by variation of donor/acceptor strengths. One simple test of the validity of this approximation was to examine the cyanine compounds $\mathbf{4}$ and $\mathbf{5}$ where the polarization was varied both by chemical substitution and by using a point charge. First, asymmetry was induced in the normally symmetric chromophore by sequentially substituting hydrogen atoms by fluorine atoms upon the methyl groups at one end of the molecule. Increases in bond length alternation from the initially bond equivalent, symmetrical cyanine were observed as the number of fluorines in the molecule increased. Second, increased bond length alternation was observed as a single Sparkle of negative charge was brought closer to the positively charged cyanine as shown in Figure 3 . As can be observed in Figure 4, when the hyperpolarizabilities for these two types of asymmetric cyanines were plotted versus bond length alternation, the resulting curves were qualitatively similar. Although the two curves were not superimposable, the corresponding maxima, minima and zero crossings were within $0.01 \AA$ of each other. Inasmuch as our goal is to explore the relationship between chemical structure and molecular hyperpolarizabilities, it was encouraging to find that polarization by a point charge closely approximates that induced by chemical substitution, especially given the diversity of the two approaches in this example.
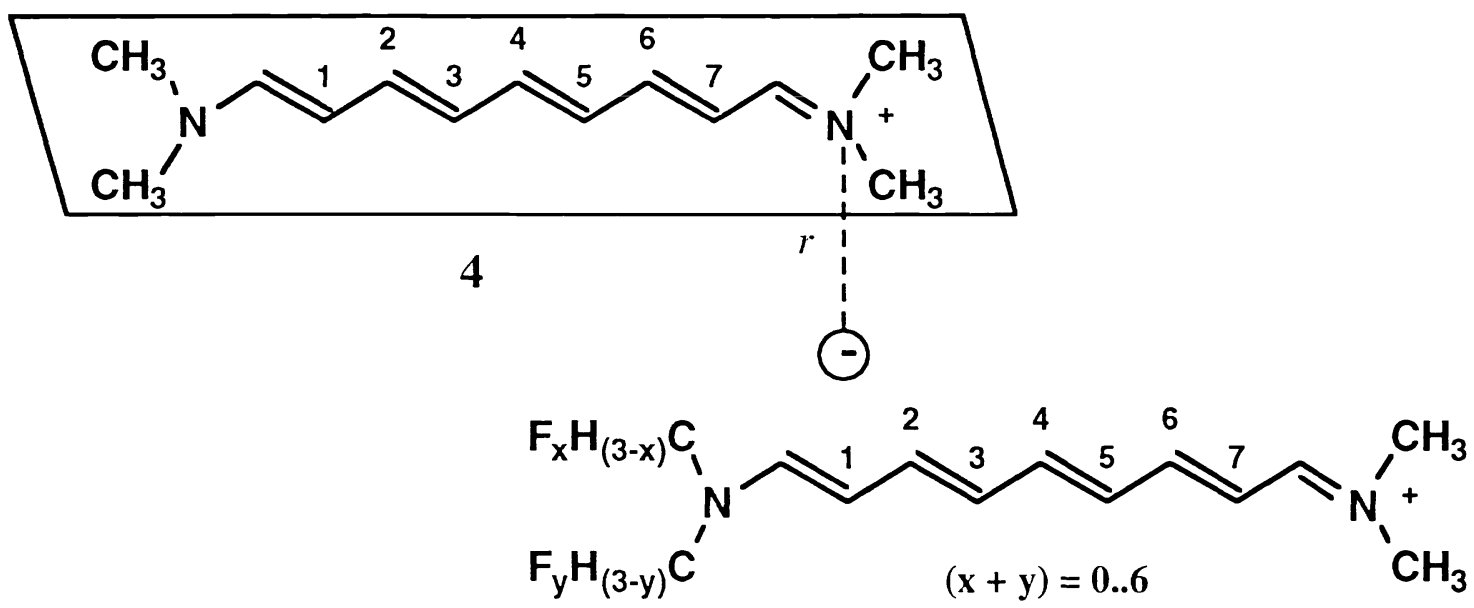

5

Figure 3. The structures of molecules 4 and 5. For each, bond length alternation is defined as $B L A=\left(r\left(C_{2}-C_{3}\right)+r\left(C_{4}-C_{5}\right)+r\left(C_{6}-C_{7}\right)\right) / 3-\left(r\left(C_{1}-C_{2}\right)+r\left(C_{3}-C_{4}\right)+r\left(C_{5}-C_{6}\right)\right) / 3$. In 4, a single Sparkle is brought up to a positively charged, symmetric cyanine. Increases in bond length alternation are observed and reported in Figure 4. For 5, increases in bond length alternation are observed as more hydrogen atoms are replaced by fluorine atoms.

Compounds 1 through 3 all contain similar donors and acceptors, but have important topological differences, leading to differing degrees of aromaticity for the 

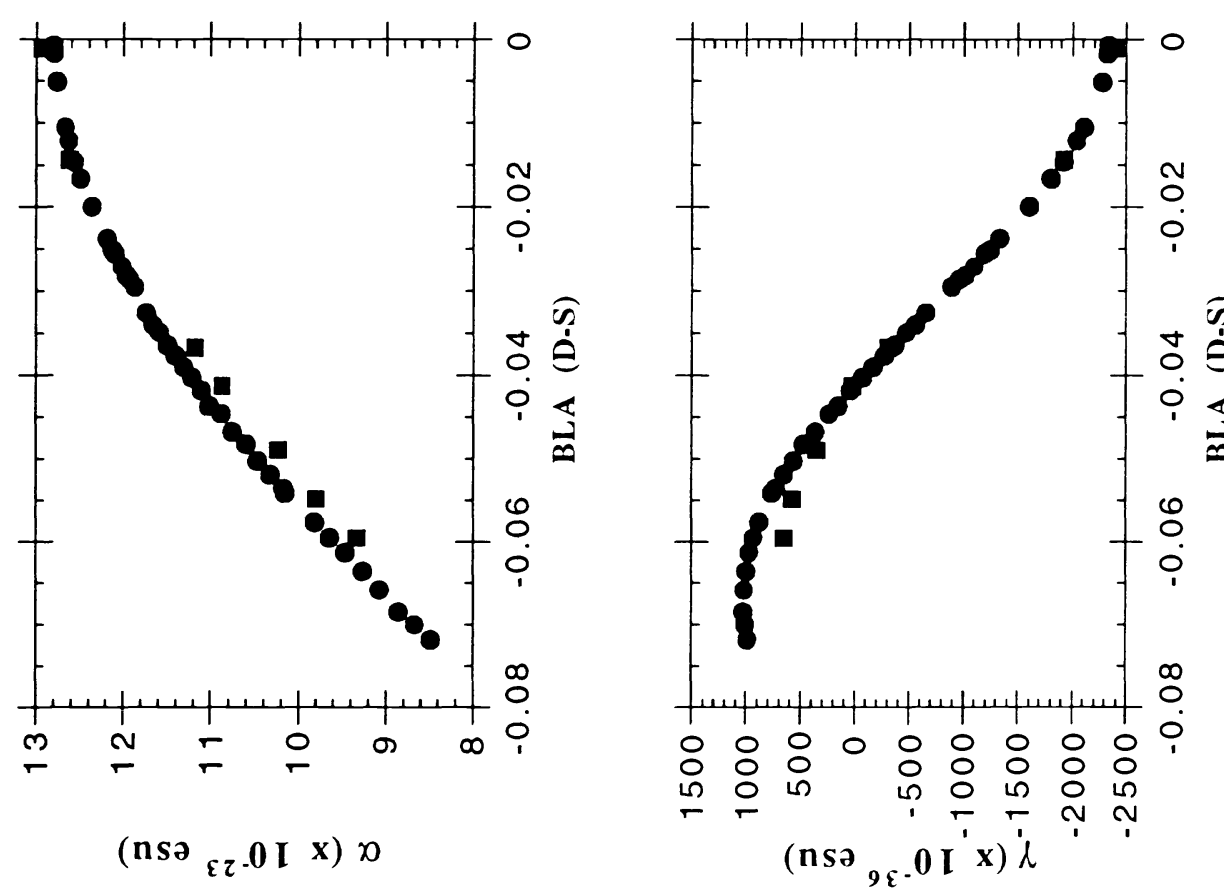

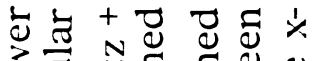

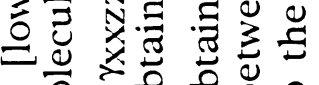
in $\div+\frac{0}{0}+\frac{0}{0}+0$

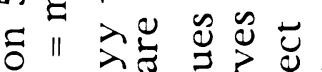

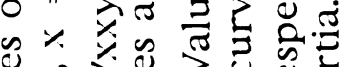

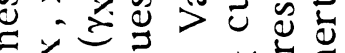
E

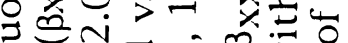
누워

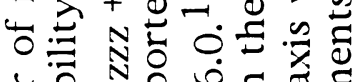

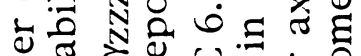

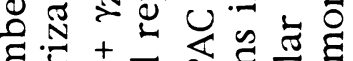

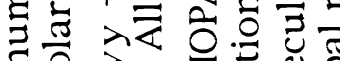

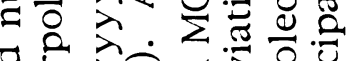

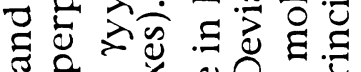
六 + 不泀的尘

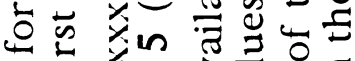

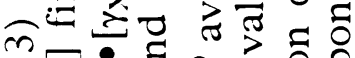

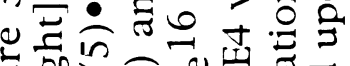
$\exists .00$ 四

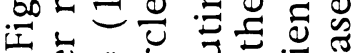
ป 11.5 $\exists$ 는

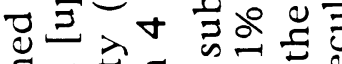

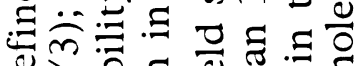
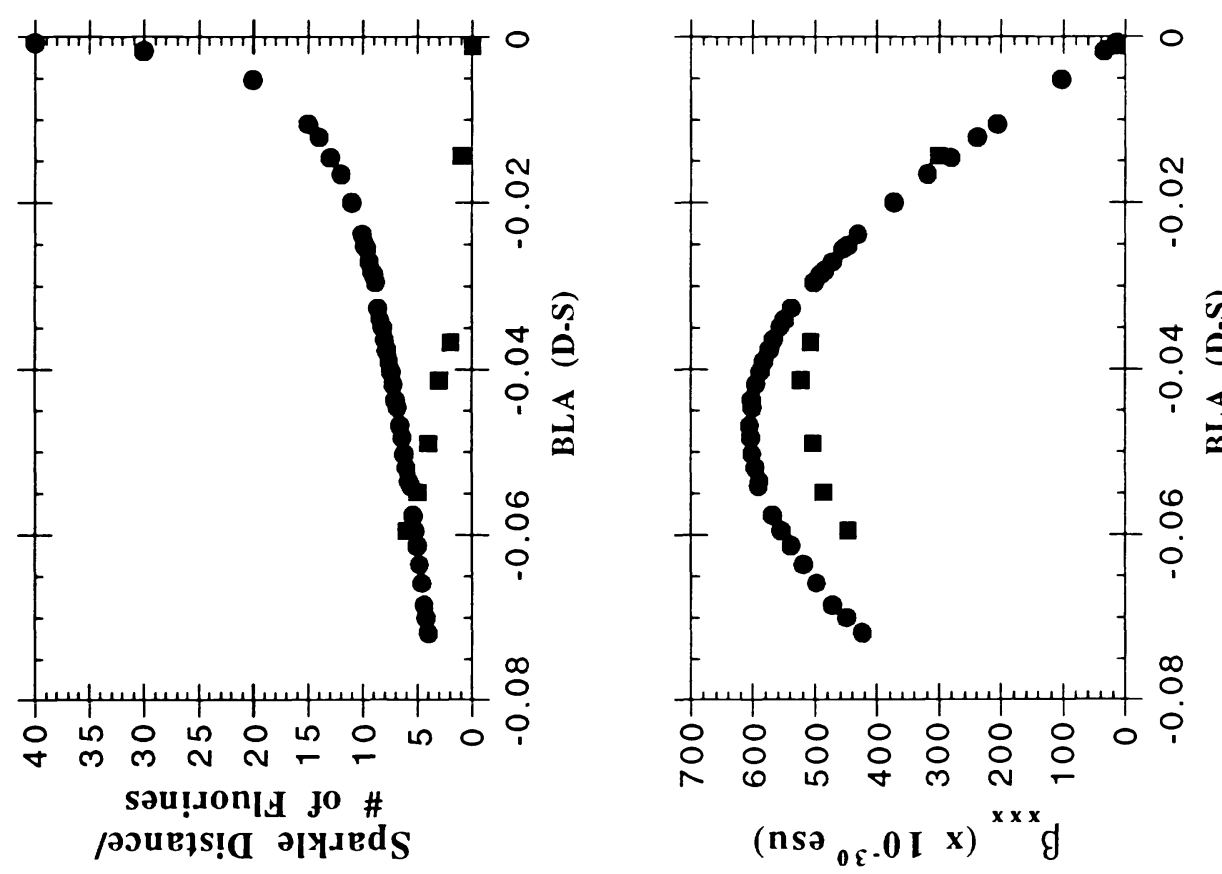

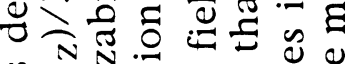
\% Nㅗㄴ $5+\frac{\sigma}{\Xi} \Xi$ 자은 ชิ

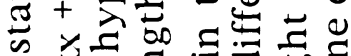

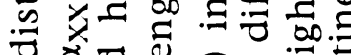

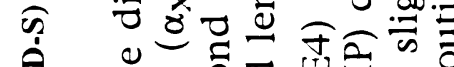

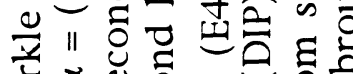

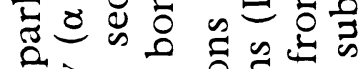
के $\lambda$ च

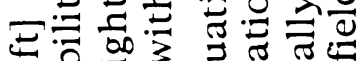
은

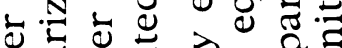
สิ

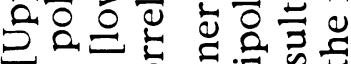
$\rightarrow$ 0 0

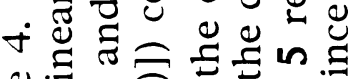
$0 \equiv$

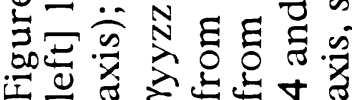


neutral canonical resonance forms. In the absence of Sparkles, the optimized geometries for 1 through 3 exhibit neutral, bond length alternated structures consistent with what would be expected in the absence of any stabilization of charge-separation (e.g. the gas phase). In 1 through $\mathbf{3}$, the double bonds not part of aromatic rings (e.g. $\mathrm{C}_{1}-\mathrm{C}_{2}$ in 1 and $2, \mathrm{C}_{2}-\mathrm{C}_{3}$ in 3 , Figure 2$)$ smoothly change from short $(\approx 1.36 \AA)$ to long $(\approx$ $1.43 \AA$ ) and the non-ring single bonds (e.g. $\mathrm{C}_{2}-\mathrm{C}_{3}$ in 1 and $2, \mathrm{C}_{1}-\mathrm{C}_{2}$ in 3 , Figure 2) change from long $(\approx 1.43 \AA)$ to short $(\approx 1.36 \AA)$ as the Sparkles move closer to the end groups of the molecule. At the same time, the ground-state dipole moments of each increase smoothly. Though molecule 1 in the gas phase is calculated to exhibit a neutral, bond alternated structure, there is experimental evidence that, in solution, the molecule is biased towards the zwitterionic structure. For example, 1 exhibits negative solvatochromism ${ }^{17}$ and a negative $\beta^{18}$ consistent with a larger ground- than excitedstate dipole moment (Figure $1 \mathrm{~b}$, right). Crystal structures of $\mathrm{Me}_{2} \mathrm{~N}-(\mathrm{CH}=\mathrm{CH}) 3-\mathrm{CHO}^{19}$ (average $\mathrm{r}(\mathrm{C}=\mathrm{C})=1.356 \AA, \mathrm{r}(\mathrm{C}-\mathrm{C})=1.416 \AA, \Delta=0.06 \AA)$ and $\mathrm{Me}_{2} \mathrm{~N}-(\mathrm{CH}=\mathrm{CH})_{3}-\mathrm{CHC}(\mathrm{CN})_{2}{ }^{19}$ (average $\mathrm{r}(\mathrm{C}=\mathrm{C})=1.376 \AA, \mathrm{r}(\mathrm{C}-\mathrm{C})=1.381 \AA, \Delta<0.01 \AA$ ) provide direct evidence for decreased bond length alternation in simple donor-acceptor polyenes relative to unsubstituted polyenes (average $\mathrm{r}(\mathrm{C}=\mathrm{C})=1.340 \AA, \mathrm{r}(\mathrm{C}-\mathrm{C})=1.445 \AA, \Delta=0.11 \AA$ from the crystal structure of 1,3,5,7-octatetraene 20 and diphenyl-1,3,5,7-octatetraene ${ }^{2}$ ). Stilbenes contain benzene rings that lose aromaticity upon charge separation and are expected to enforce bond length alternation. For example, in the crystal of 2-methoxy$4^{\prime}$-nitro-stilbene, $\mathrm{r}(\mathrm{C}=\mathrm{C})=1.31 \AA$, average $\mathrm{r}(\mathrm{C}-\mathrm{C})=1.47 \AA, \Delta=0.16 \AA .^{22}$ Using Sparkles, 1 through 3 could be polarized into a configuration in which there was no bond length alternation, corresponding to a cyanine-like molecule. At this point, the two limiting resonance forms, displayed for $\mathbf{1}, \mathbf{2}$ and $\mathbf{3}$, have approximately the same contribution to the ground- and excited-states. For 1, 2 and 3, zero bond length alternation (see Figure 2) occurred at Sparkle distances of $7.6 \AA, 5.6 \AA$ and $4.6 \AA$ (corresponding to an average external electric field along the molecular axis of $1.8 \times 10^{9} \mathrm{~V} / \mathrm{m}, 2.5 \times 10^{9} \mathrm{~V} / \mathrm{m}$ and $3.4 \mathrm{x}$ $10^{9} \mathrm{~V} / \mathrm{m}$ respectively (Figure 5). The gain in aromaticity upon charge separation in 1 is clearly seen to stabilize the zwitterionic form at a given Sparkle distance, accounting for this offset. Likewise, the loss in aromaticity upon charge separation in 3 requires a greater electric field to polarize the molecule to this degree (Figure 5).

The basic shapes of the curves for $\mu, \alpha, \beta$ and $\gamma$ were the same for the three molecules when these quantities were observed versus bond length alternation. The peak values of linear and nonlinear polarizabilities of $\mathbf{2}$ and $\mathbf{3}$ were greater than those of 1 , most likely because the former two molecules are longer. ${ }^{23}$ Our calculations show $\alpha$ to be peaked for $\mathbf{1}$ through $\mathbf{3}$ within $0.01 \AA$ of the cyanine limit (zero bond length alternation), consistent with the observation that cyanines are the most polarizable class of linear conjugated organic molecules examined to date. ${ }^{24}$ At this point, however, large but approximately equal ground- and excited-state dipole moments are expected and $\beta$, which is proportional to their difference, is approximately zero, consistent with the predictions of the two-state model (Equation 1). Indeed, the shapes of $\beta$ curves are consistent with those previously predicted based on a four orbital calculation. ${ }^{1}$ The detailed dependence of $\gamma$ as a function of ground-state polarization, its correlation with 


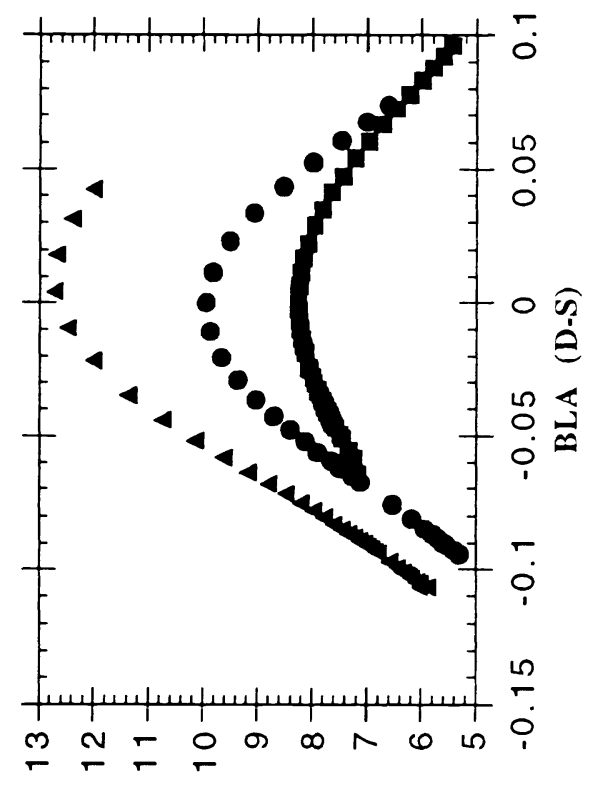

$\operatorname{lns}_{\varepsilon \tau-0 I}$ x) $)$
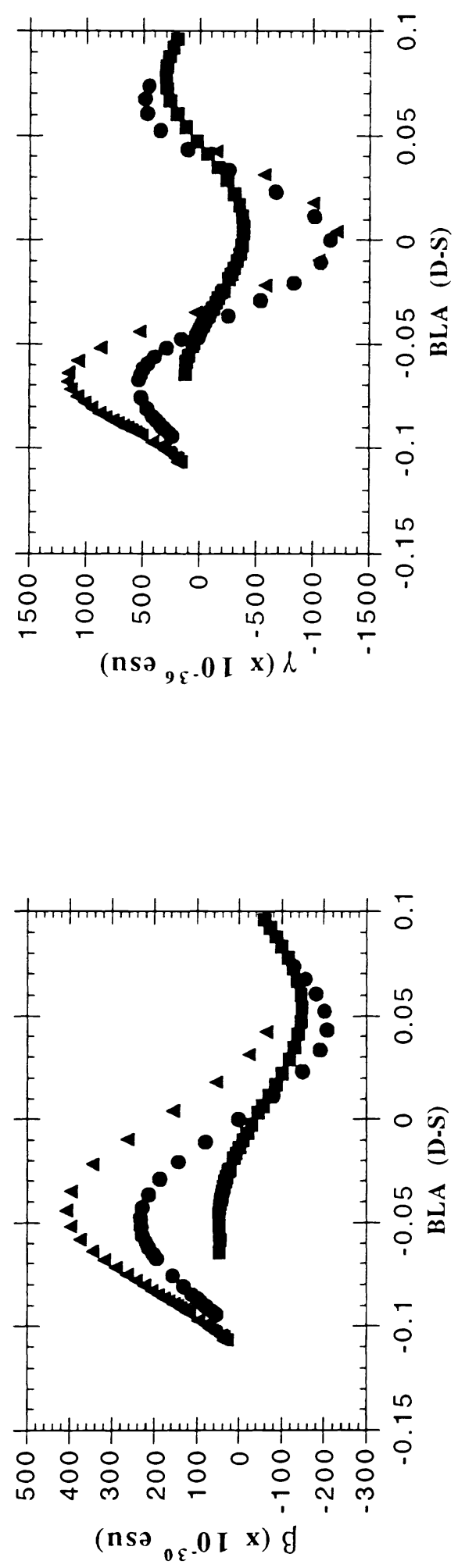

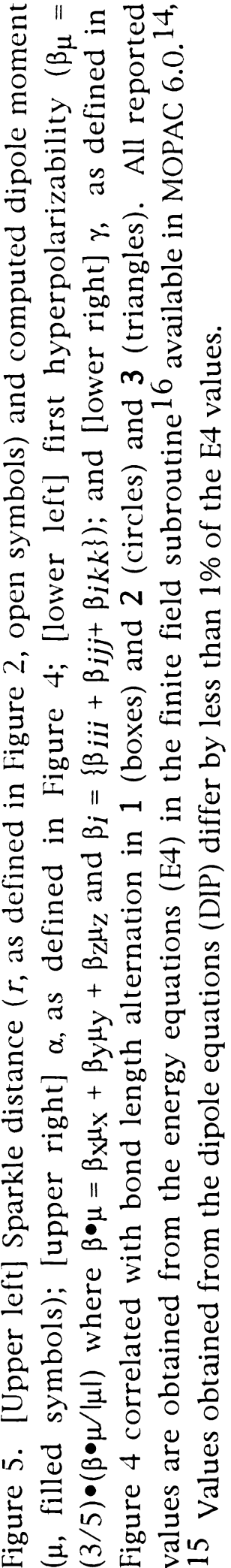


bond length alternation, or its behavior as compared to $\alpha$ and $\beta$ has not to our knowledge been previously described. There are several important structure/property predictions that result from our analysis. First, the $\gamma$ curves for all three molecules exhibit negative peaks at zero bond length alternation and positive peaks at both positive and negative bond length alternation. Second, the negative peak values are somewhat larger than the positive peak values in magnitude. Third, 2 , at its positive peak, has a 2- to 4-fold enhancement of $\gamma$ relative to that of a nonpolarized (unsubstituted) polyene of comparable length $(\gamma=1,078,410$ a.u. for 2 vs. 241,012 a.u. for a 10 carbon polyene and 489,040 a.u. for a 12 carbon polyene). ${ }^{25}$ Fourth, $\gamma$ peaks at larger absolute values of bond length alternation than $\beta$ and, in fact, when $\beta$ is peaked, $\gamma$ is roughly zero. Qualitatively, the behavior of $\gamma$ predicted here is consistent with that predicted by a three term model previously derived from perturbation theory26-30 in which:

$$
\gamma \propto-\left(\frac{\mu_{\mathrm{ge}}^{4}}{E_{\mathrm{ge}}^{3}}\right)+\left(\frac{\mu_{\mathrm{ge}}^{2} \mu_{\mathrm{e}^{\prime}}^{2}}{E_{\mathrm{ge}}^{2} E_{\mathrm{ge}}}\right)+\left(\frac{\mu_{\mathrm{ge}}^{2}\left(\mu_{\mathrm{ee}}-\mu_{\mathrm{gg}}\right)^{2}}{E_{\mathrm{ge}}^{3}}\right)
$$

where $g$ and $e$ are labels as in the two-state expression above, and $e^{\prime}$ labels a second excited-state. When the first term is dominant, negative $\gamma$ results as is so for cyanines, ${ }^{31}$ and related squarylium dyes. ${ }^{29}$ For centrosymmetric polyenes, the second term dominates and the third term is zero resulting in positive $\gamma .{ }^{31,32}$ Enhanced $\gamma$ in donor/acceptor diphenyl polyenes relative to their centrosymmetric parent compounds has also been observed. ${ }^{23}, 33$ For these types of polarized polyenes, the third term will enhance $\gamma$, however, since $\left(\mu_{\mathrm{e}} \mathrm{e}^{\left.-\mu_{\mathrm{gg}}\right)}\right.$ exhibits a peak as bond length alternation ${ }^{1}$ decreases and goes through zero at roughly zero bond length alternation, its contribution will be small near the cyanine limit. Furthermore, since $\left(\mu_{\mathrm{ge}}^{4} / E_{\mathrm{ge}}^{3}\right)$ also increases with decreasing ground-state bond length alternation ${ }^{1}$ and is of opposite sign to the third term, this simple analysis of the perturbation theory expression can explain the peaked $\gamma$ curves that we calculate using MOPAC.

These external electric field (Sparkle distance) dependent calculations of hyperpolarizabilities support the hypothesis that bond length alternation is a key structural paremeter to examine in the development of structure/property relationships for $\alpha, \beta$ and $\gamma$. The role of aromaticity in determining the ground-state structure and polarizability is clearly illustrated in the comparison of $\mathbf{1}, \mathbf{2}$ and $\mathbf{3}$. Most organic compounds with predominantly aromatic ground-states will have greater than $0.1 \AA$ bond length alternation in the polyene portion of the molecule. We therefore predict that for the class of end-capped polymethine dyes, those with reduced aromaticity in the neutral canonical resonance form can result in the largest nonresonant $\beta$ and $\gamma$.

Where experimental and theoretical comparisons are available, there is good qualitative agreement with the results of these electric field dependent calculations of hyperpolarizability, suggesting that this method can be used generally to map out structure/property relationships for different molecular topologies. We observe that 
the $\beta$ curve for 2 qualitatively has a very similar appearance to the derivative of its $\alpha$ curve, and, similarly, its $\gamma$ curve looks much like the derivative of its $\beta$ curve. These relationships are intriguing and could have important implications if they prove to be general. Finally, the marked sensitivity of $\alpha, \beta$ and $\gamma$ upon external electric field underscores the need to account for medium dependent changes in both electronic and geometric structure if one is to develop an understanding of structure/property relationships of organic molecules in condensed phases.

\section{FIRST HYPERPOLARIZABILITIES OF POLARIZED POLYENES}

Although there have been several computational studies of the first hyperpolarizability $(\beta)$ of the simplest organic donor-acceptor chromophores, the polyenes, ${ }^{34}, 35$ these molecules have not yet been studied experimentally. Donoracceptor polyenes containing between 3 and 11 conjugated atoms have been purchased or synthesized as described in the literature, $8,36,37$ and their dipole moment $(\mu)$ and $\beta$ have been measured by EFISH with $1.907 \mu \mathrm{m}$ fundamental radiation in $\mathrm{CHCl}_{3} .^{23}, 33$ Values of $\beta$ and $\mu$ are presented in Table 1 as well as the optical absorption maximum $\left(\lambda_{\max }\right), \beta(0)$ corrected for dispersion using a two-state model, and $(\mu \bullet \beta)$, the dot product of $\beta$ with $\mu$, a quantity of interest in poled polymers applications. These chromophores possess large dipole moments (9-10 Debye for the dicyanovinyl acceptor) and large $\mu \bullet \beta$ values.

$\beta(0)$ values of the simple polyenes series are increased compared to polyenes containing an $\alpha$-phenyl ring. ${ }^{23}$ It can be rationalized that the former set of molecules has a more optimal bond length alternation to maximize $\beta$. For the $\alpha$-phenyl polyene series, the loss of aromaticity accompanying charge separation will diminish the contribution of the charge-separated resonance form to the ground-state wave function and, as such, the $\alpha$-phenyl polyene series may have too much bond length alternation to maximize $\beta$. In contrast, the simple donor-acceptor polyenes, unhampered by aromaticity, will exhibit greatly reduced bond length alternation, as noted earlier.

Although a dicyanovinyl group is generally considered to be a better acceptor than an aldehyde, the $\beta(0)$ values of the polyene aldehyde chromophores are greater than those for the dicyanovinyl chromophores with the same number of atoms between the amine nitrogen and the last heteroatom of the acceptor group. Two possible explanations for these results are: 1) there is ineffective electron delocalization into the cyano groups (evidenced by the typically short $\mathrm{C} \equiv \mathrm{N}$ bond) thereby reducing the effective conjugation length of the molecule and 2) in the relatively polar solvent, in which the EFISH experiment was performed, $\left(\mathrm{CHCl}_{3}\right)$ the dicyanovinyl is a sufficiently strong acceptor that the bond length alternation (and asymmetric polarizability) is reduced below the point at which $\beta$ is maximized. Since solvent polarity can be used to tune the degree of ground-state bond alternation in these systems, $6,11,38$ solvent 
dependent EFISH measurements of $\beta$ for the polyene aldehyde and dicyanovinyl series will be used to elucidate the origin of these unusual results.

The data in Table 1 can be fit to a function of the form $\beta(0) \propto N^{m}$, where $N$ is the number of conjugated multiple bonds in the molecule (including the carbon heteroatom bond as one and the phenyl group as two). The exponent, $m$, lends insight into the chain length dependence of $\beta(0)$. The values of $\mathrm{m}$ for the simple polyene aldehydes and dicyanovinyl series are 3.2 and 5.4 respectively. For comparison, $\alpha$-phenyl polyene aldehyde and dicyanovinyl series ${ }^{23}$ gave $\mathrm{m}=3.5$ and 3.6 respectively. The exponent observed for the dicyanovinyl polyene series is larger than those for the other series reported here and for any other class of organic molecules in the literature. Furthermore, although solutions of the amino polyene aldehydes are not stable to air and light for long periods of time, the dicyanovinyl compounds are relatively stable under ambient conditions. Thus, given their large $\mu \bullet \beta$ values, low molecular volume and reasonable solubility, the latter series could be of interest for poled polymer applications.

Table I. Linear and non-linear optical properties of donor-acceptor polyenes of the form $\mathrm{D}-(\mathrm{CH}=\mathrm{CH})_{\mathrm{n}}-\mathrm{A}$.

\begin{tabular}{|c|c|c|c|c|c|c|c|c|}
\hline $\mathrm{D}$ & A & $\mathrm{n}$ & $\begin{array}{c}\text { \# } \\
\text { Conjugated } \\
\text { Atoms } \\
\end{array}$ & $\begin{array}{l}\lambda_{\max } \\
(\mathrm{nm})\end{array}$ & $\begin{array}{c}\mu \times 10^{-18} \\
(\text { esu })\end{array}$ & $\begin{array}{c}\beta \times 10^{-30} \\
(\mathrm{esu})\end{array}$ & $\begin{array}{c}\beta(0) \times 10^{-30} \\
(\mathrm{esu})\end{array}$ & $\begin{array}{c}\mu \beta \times 10^{-48} \\
(\text { e s u })\end{array}$ \\
\hline $\mathrm{Me}_{2} \mathrm{~N}$ & $\mathrm{CHO}$ & 0 & 3 & $<240$ & 3.5 & 0.5 & 0.5 & 1.8 \\
\hline $\mathrm{Me}_{2} \mathrm{~N}$ & $\mathrm{CHO}$ & 1 & 5 & 284 & 6.3 & 3.3 & 2.9 & 21 \\
\hline $\mathrm{Et}_{2} \mathrm{~N}$ & $\mathrm{CHO}$ & 2 & 7 & 363 & 6.5 & 20 & 16.5 & 130 \\
\hline $\mathrm{Me}_{2} \mathrm{~N}$ & $\mathrm{CHO}$ & 3 & 9 & 422 & 6.9 & 53 & 41 & 366 \\
\hline $\mathrm{Me}_{2} \mathrm{~N}$ & $\mathrm{CH}=\mathrm{C}(\mathrm{CN})_{2}$ & 0 & 5 & 352 & 7.6 & 1.0 & 0.9 & 7.6 \\
\hline $\mathrm{Me}_{2} \mathrm{~N}$ & $\mathrm{CH}=\mathrm{C}(\mathrm{CN})_{2}$ & 1 & 7 & 374 & 8.9 & 6.1 & 5.0 & 54 \\
\hline $\mathrm{Et}_{2} \mathrm{~N}$ & $\mathrm{CH}=\mathrm{C}(\mathrm{CN})_{2}$ & 2 & 9 & 476 & 10.7 & 45 & 32 & 482 \\
\hline $\mathrm{Me}_{2} \mathrm{~N}$ & $\mathrm{CH}=\mathrm{C}(\mathrm{CN})_{2}$ & 3 & 11 & 550 & 9.9 & 211 & 129 & 2089 \\
\hline $\mathrm{Me}_{2} \mathrm{NC}_{6} \mathrm{H}_{4}$ & $\mathrm{CHO}$ & 0 & 7 & 326 & 5.1 & 6.3 & 5.4 & 32 \\
\hline $\mathrm{Me}_{2} \mathrm{NC}_{6} \mathrm{H}_{4}$ & $\mathrm{CHO}$ & 1 & 9 & 384 & 5.6 & 30 & 24 & 168 \\
\hline $\mathrm{Me}_{2} \mathrm{NC}_{6} \mathrm{H}_{4}$ & $\mathrm{CH}=\mathrm{C}(\mathrm{CN})_{2}$ & 0 & 9 & 420 & 7.8 & 32 & 25 & 250 \\
\hline $\mathrm{Me}_{2} \mathrm{NC}_{6} \mathrm{H}_{4}$ & $\mathrm{CH}=\mathrm{C}(\mathrm{CN})_{2}$ & 1 & 11 & 486 & 8.4 & 82 & 57 & 689 \\
\hline
\end{tabular}




\section{CONCLUSIONS}

The theoretical and experimental results reported here suggest that, by the correct choice of the degree of aromatic-quinonal character in the ground-state wave function, it should be possible to strike the optimal compromise for the relative energetics of the neutral and CT resonance forms to maximize $\alpha, \beta$ or $\gamma$. It has been shown that bond length alternation is a key, structurally observable parameter that can be correlated with hyperpolarizabilities and is extremely relevant to the optimization of molecules and materials. $\quad \beta$ is predicted to peak at $\sim 0.04 \AA$ of bond length alternation. Likewise, $\gamma$ is predicted to have a positive peak at $\sim 0.06 \AA$ of bond length alternation and a negative peak of larger magnitude at zero bond length alternation. Most molecules currently under investigation are either polyenes, polyaromatics, or some combination of the two and have $\sim 0.1 \AA$ or more bond length alternation. This study suggests that such molecules are too bond length alternated to maximize $|\beta|$ or $|\gamma|$.

\section{ACKNOWLFDGMENTS}

The work in this paper was performed, in part, at the Center for Space Microelectronics Technology, Jet Propulsion Laboratory (JPL), California Institute of Technology under contract with the National Aeronautics and Space Administration (NASA). The work was sponsored by the Defense Advanced Research Projects Agency through a contract administered by the Air Force Office of Scientific Research and the Strategic Defense Initiative Organization Innovative Science and Technology Office. Support from the National Science Foundation (Grant CHE-9106689) and Air Force Office of Scientific Research (Grant F49620-92-J-0177) is also gratefully acknowledged. CBG thanks the JPL directors office for postdoctoral fellowship.

\section{REFERENCES AND FOOTNOTES}

(1) Marder, S. R.; Beratan, D. N.; Cheng, L.-T. Science 1991, 252, 103-106.

(2) Oudar, J. L.; Chemla, D. S. J. Chem. Phys. 1977, 66, 2664-2668.

(3) Levine, B. F.; Bethea, C. G. J. Chem. Phys. 1977, 66, 1070.

(4) Lalama, S. J.; Garito, A. F. Phys. Rev. A 1979, 20, 1179.

(5) Paley, M. S.; Harris, J. M.; Looser, H.; Baumert, J. C.; Bjorklund, G. C.; Jundt, D.; Twieg, R. J. J. Org. Chem. 1989, 54, 3774-3778.

(6) Nolte, K. D.; Dähne, S. Adv. Mol. Relax. Interact. Proc. 1977, 10, 299-329.

(7) Benson, H. G.; Murrell, J. N. J. Chem. Soc., Faraday Trans. 2 1972, 68, 137-143.

(8) Scheibe, P.; Schneider, S.; Dörr, F.; Daltrozzo, E. Ber. Buns. Ges. 1976, 80, 630-638.

(9) Schneider, S. Ber. Buns. Ges. 1976, 80, 218-222.

(10) Radeglia, R.; Engelhardt, G.; Lippmaa, E.; Pehk, T.; Nolte, K. D.; Daehne, S. Org. Magn. Resonance 1972, 4, 571-6.

(11) Radeglia, R.; Dähne, S. J. Mol. Struct. 1970, 5, 399-411. 
(12) Radeglia, R.; Steiger, T. J. Prakt. Chem. 1991, 333, 505-8.

(13) Luzhkov, V.; Warshel, A. J. Am. Chem. Soc. 1991, 113, 4491-4499.

(14) Stewart, J. J. P. J. Comput. Chem. 1989, 10, 209-220.

(15) Stewart, J. J. P. J. Comput. Chem. 1989, 10, 221-264.

(16) Kurtz, H. A.; Stewart, J. J. P.; Dieter, K. M. J. Comput. Chem. 1990, 11, 82-87.

(17) Brooker, L. G. S.; Keyes, G. H.; Heseltine, D. W. J. Am. Chem. Soc. 1951, 73, 53505356.

(18) Levine, B. F.; Bethea, C. G.; Wasserman, E.; Leenders, L. J. Chem. Phys. 1978, 68, 5042-5045.

(19) Marder, S. R., et al. Submitted.

(20) Baughman, R. H.; Kohler, B. E.; Levy, I. J.; Spangler, C. Synth. Met. 1985, 11, 37 52.

(21) Drenth, W.; Wiebenga, E. H. Acta Cryst. 1955, 8, 755-760.

(22) Grubbs, R. B.; Marder, S. R.; Perry, J. W.; Schaefer, W. P. Chem. Mater. 1991, 3, 3 4.

(23) Cheng, L.-T.; Tam, W.; Marder, S. R.; Steigman, A. E.; Rikken, G.; Spangler, C. W. J. Phys. Chem. 1991, 95, 10643-10652.

(24) Dähne, S.; Nolte, K. D. J. Chem. Soc., Chem. Commun 1972, 1056-1057.

(25) Kurtz, H. A. Int. J. Quant. Chem. Quant. Chem. Symp. 1990, 24, 791-798.

(26) Garito, A. F.; Heflin, J. R.; Wong, K. Y.; Zamani-Khamiri, O. In Organic Materials for Non-linear Optics: Royal Society of Chemistry Special Publication No. 69; Hann, R. A.; Bloor, D. Ed.; Royal Society of Chemistry, Burlington House: London, 1989; pp 16-27.

(27) Dirk, C. W.; Kuzyk, M. G. In Materials for Nonlinear Optics: Chemical Perspectives; Marder, S. R.; Sohn, J. E.; Stucky, G. D. Ed.; ACS Symposium Series Vol. 455; American Chemical Society: Washington, DC, 1991; pp 687-703.

(28) Pierce, B. M. Proc. SPIE 1991, 1560, 148-161.

(29) Dirk, C. W.; Cheng, L.-T.; Kuzyk, M. G. Int. J. Quant. Chem. 1992, 43, 27-36.

(30) Kuzyk, M. G.; Dirk, C. W. Phys. Rev. A 1990, 41, 5098-5109.

(31) Stevenson, S. H.; Donald, D. S.; Meredith, G. R. In Nonlinear Optical Properties of Polymers; Heeger, A. J.; Orenstein, J.; Ulrich, D. R. Ed.; Materials Research Society Symposium Proceedings Vol. 109; Materials Research Society: Pittsburgh, 1988; pp 103-108.

(32) Perry, J. W.; Steigman, A. E.; Marder, S. R.; Coulter, D. R.; Beratan, D. N.; Brinza, D. E.; Klavetter, F. L.; Grubbs, R. H. Proc. SPIE 1988, 971, 17-24.

(33) Cheng, L.-T.; Tam, W.; Stevenson, S. H.; Meredith, G. R.; Rikken, G.; Marder, S. R. J. Phys. Chem. 1991, 95, $10631-10643$.

(34) Li, D.; Ratner, M. A.; Marks, T. J. J. Am. Chem. Soc. 1988, 110, 1707-1715.

(35) Morley, J. O.; Docherty, V. J.; Pugh, D. J. Chem. Soc., Perkin Trans. II 1987, 1351 1355.

(36) Malhotra, S. S.; Whiting, M. C. J. Chem. Soc. 1960, 3812-3822.

(37) Becher, J. Synthesis 1980,8, 589-612.

(38) Dähne, S.; Nolte, K.-D. Acta Chim. Acad. Sci. Hung. 1978, 97, 147-157. 